Classification

Physics Abstracts

$62.20-62.40-63.20$

\title{
A lattice-dynamics model of an oscillating screw dislocation
}

\author{
N. E. Glass (*) \\ Institut de génie atomique, Swiss Federal Institute of Technology (EPFL), CH-1015 Lausanne, Switzerland
}

(Reçu le 29 décembre 1982, révisé le 14 février 1983, accepté le 16 février 1983)

\begin{abstract}
Résumé. - L'idée de la « force-source », ou force de Kanzaki, pour une dislocation vis statique est généralisée au cas d'une dislocation mobile dans un réseau harmonique cubique simple (modèle dit du " snapping-bond "); le modèle est utilisé avec la fonction de Green relative aux phonons, pour obtenir les expressions donnant les déplacements atomiques et la dissipation d'énergie moyenne dans le temps, c'est-à-dire, le taux de transfert d'énergie de la dislocation mobile aux vibrations de réseau. Dans le cas où la dislocation oscille de manière harmonique en fonction du temps, une expression pour la dissipation d'énergie, évaluée à l'ordre le plus bas en fréquence, est obtenue qui se réduit, pour les fréquences de l'ordre du mégahertz ou plus basses, à une somme finie et une intégrale finie. On présente ensuite comment cette méthode pourrait être généralisée pour décrire un réseau qui contient une faible concentration $(c)$ de défauts ponctuels répartis aléatoirement, et on montre comment le changement de la dissipation d'énergie est proportionnel à $c$, à l'ordre le plus bas. La méthode développée ici est telle qu'on peut la généraliser, en principe, d'une façon non ambigu au cas d'une force atomique non linéaire, ou anharmonique, ainsi que d'un amortissement dû aux phonons et dépendant de la température.
\end{abstract}

\begin{abstract}
The concept of the Boyer-Hardy source-force, or Kanzaki force, for a static screw dislocation is extended to describe a moving dislocation - in a simple cubic, nearest neighbour, harmonic lattice (snapping-bond model) and together with the phonon Green's function is used to derive expressions for the atomic displacements and timeaveraged energy disspation, viz. the rate of energy transfer from the moving dislocation to the lattice vibrations. For the case in which the dislocation oscillates harmonically in time, an expression for the energy dissipation is derived which, for megahertz frequencies and lower, is then reduced to a finite sum and finite integration, and is evaluated to lowest (zeroth) order in the frequency. The way in which the method may be extended to describe a lattice containing a low concentration $c$ of point defects in a random array is then outlined, and it is shown how the change in energy dissipation, to lowest order, is proportional to $c$. The method developed here is such that it can, in principle, be further generalized in a completely unambiguous fashion, to allow for a non-linear or anharmonic atomic force-law in the dynamic source-force of the dislocation as well as a temperature dependent phonon damping in the Green's function.
\end{abstract}

\section{Introduction.}

The need for a lattice or atomistic description of dislocations, both static and dynamic, has been long recognized. In the case of dislocation statics, the inadequacy of linear elastic continuum-theory to describe the core structure and energy was immediately apparent because of the infinities it yields on the dislocation line. This problem was first dealt with in a purely lattice model by Maradudin, [1] who considered a screw dislocation within a rectangular lattice of atomic rows, interacting by nearest-neigh-

(*) Present address : Department of Physics, University of California, Irvine, California 92717, U.S.A. bour harmonic forces. By introducing a source-force representing the broken and re-attached atomic bonds across a semi-infinite cut-plane, Maradudin calculated the atomic displacements, along the dislocation direction, necessary for static equilibrium. This method was extended by Boyer and Hardy [2] to allow for relaxation of the lattice in the plane perpendicular to the dislocation line. These authors expressed the statics problem explicitly in terms of the static lattice Green's function and a general source-force or Kanzaki force, in such a way as to allow for the treatment of a screw dislocation in an arbitrary Bravais lattice, in which atoms may interact at any distance via a central pairwise force.

In the case of dislocation dynamics, the need for 
lattice models has been demonstrated both experimentally and theoretically. This need is particularly evident in phenomena involving dislocations moving through a crystal containing point defects. It has recently been shown [3] that models based on continuum theory that treat the dislocations as a vibrating string with viscous damping (Koehler-Granato-Lucke model) do not properly describe the so-called peakingeffect - the increase in internal friction, measured under longitudinal vibrations from $10^{2}$ to $10^{5} \mathrm{~Hz}$, during the irradiation of certain fcc metals $(\mathrm{Cu}, \mathrm{Ag}, \mathrm{Al})$, originally seen by Simpson, Sosin et al. [4, 5]. A simple phenomenological model which accounts for all the observed characteristics of this peakingeffect was recently proposed [6]. This model does not employ continuum theory's viscous damping : the increase in energy dissipation due to the oscillating dislocation is attributed to the increase in the energy released by each broken atomic bond, in the plane of the dislocation motion, caused by the global effect of the irradiation-induced point defects on the lattice dynamics.

The ideas in this phenomenological model are consistent with the lattice-dynamics calculation of Ishioka [7]. Ishioka, like Atkinson and Cabrera [8] before him, used a one-dimensional model of a dislocation (a Frenkel-Kontorova model), to which he added an anharmonic substrate potential and a damping of the lattice vibrations. Assuming a uniform dislocation motion, he solved numerically the equations of motion for the atoms, and found three distinct kinds of motion, corresponding to three regions of dislocation velocity. There is an intermediate velocity domain where the calculated displacements conform with continuum theory, but there also is a low velocity region of "pulsating motion" where the results look entirely different from those of continuum theory. As pointed out in reference [6], if the intermediate region corresponds to megahertz frequencies, where the vibrating-string model correctly predicts the acoustic attenuation, then the low velocity region should correspond to the submegahertz frequencies of the peaking effect, where continuum models fail to explain the experimental measurements. Finally, there is a high velocity region, where energy dissipation is due to the emission of lattice waves in agreement with the three-dimensional lattice model of screw dislocation motion of Celli and Flytzanis [9, 12].

The Celli and Flytzanis treatment of the uniform motion of a screw dislocation in a cubic lattice, with piecewise-linear nearest-neighbour interactions (the simplest of the snapping-bond models that they consider), is just a generalization of Maradudin's method : the source-term, representing the force across the cut-plane in Maradudin's equation of static equilibrium, is given a time dependence - through a step-function of the dislocation position at time $t$ - and an inertial acceleration term is added [9-12].
None of the above lattice dynamical models of dislocation motion consider a harmonically oscillating dislocation or discuss the possibility of including the effects of point defects - important considerations in understanding the internal friction of metals, particularly when irradiated or solute-hardened. The equations developed here are intended as a first step toward that end.

The present work, like that of Celli and Flytzanis, begins by generalizing from a screw-dislocation statics-equation to the lattice dynamics equation, but unlike the previous work, it does not start with the Newtonian equation of motion. Rather, here one simply ,puts the time dependence directly into the Boyer-Hardy source-term, or Kanzaki force, and replaces the inverse force-constant matrix (static Green's function) with the phonon Green's function. This is of course physically equivalent to the CelliFlytzanis snapping-bond approach. It enables one, however, to express more directly and immediately certain measurable quantities, such as the energy dissipation and hence internal friction, in terms of quantities that can be generalized in a very obvious manner, viz. the imaginary part of a Green's function that can be made to include the effects of phonon damping due to point defects or anharmonicity through the introduction of a simple self-energy.

A general expression is derived, in terms of a phonon Green's function, for the energy dissipation due to a screw dislocation oscillating harmonically in a cubic, nearest-neighbour, harmonic lattice. That expression is developed analytically for the case of a perfect-lattice Green's function, and in the low frequency limit is reduced, with some numerical integration, to a simple algebraic expression. Finally a brief outline of how the method can be extended to include a low concentration of random point defects is presented.

\section{The Kanzaki force, atomic displacements, and energy dissipation for a moving screw dislocation.}

In this section the Kanzaki force for a moving screw dislocation is presented and is used to express the atomic displacement field and energy dissipation.

First, consider a simple cubic lattice with an infinitely long screw dislocation parallel to the $z$-axis and intersecting the centre of a cube face (Fig. 1). The dislocation is formed from the perfect lattice by cutting all pair-wise atomic bonds that intersect the upper half $(y>0)$ of the $y z$-plane, and by then reattaching each broken bond one lattice constant down the $z$-axis (as indicated in Fig. 1, e.g., cut 4-1 and create 4-1a). In the harmonic approximation following Boyer and Hardy [2] the source-force or Kanzaki force which acts in the Cartesian direction $i$ on the atom at $\mathbf{r}^{\mathbf{m}}$ is given by :

$$
K_{i}^{m} \equiv \sum_{n, j}\left[\mathbf{r}^{m}, \mathbf{r}^{n}\right] \Phi_{i j}^{m n} b \delta_{i 3}
$$




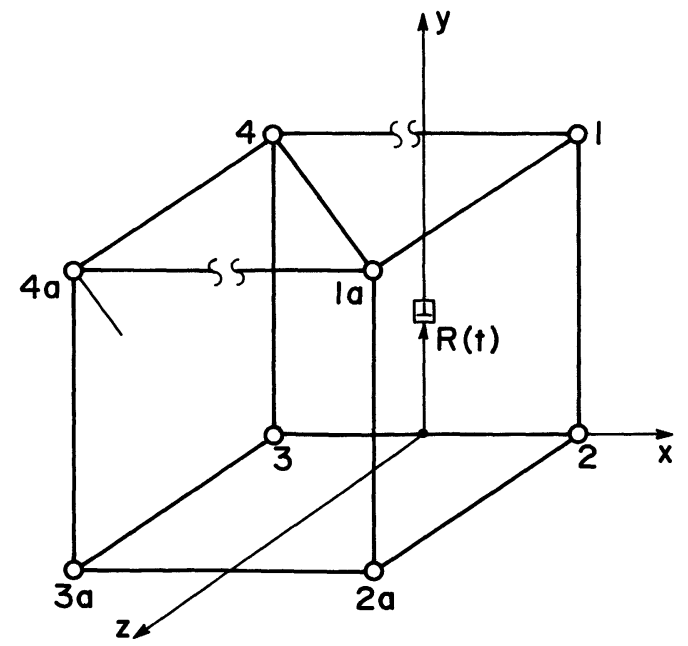

Fig. 1. - Geometry of the model considered : a simple cubic lattice containing an infinitely long screw dislocation along the $z$-axis, moving in the $y$-direction, and intersecting the $x y$-plane at the position marked $\perp$.

where $-\Phi_{i j}^{m n}$ is the harmonic force-constant (force in direction $i$ on atom $\mathbf{r}^{m}$ when atom $\mathbf{r}^{n}$ is given a unit displacement in direction $j$ ), and where

$\left[\mathbf{r}^{m}, \mathbf{r}^{n}\right]=\left\{\begin{array}{l}+1(-1), \text { if the } m-n \text { bond crosses the cut- } \\ \text { plane (but not through the dislocation line) } \\ \text { and if } m \text { is to the left (right) of } n \\ +1 / 2(-1 / 2), \text { if the } m-n \text { bond crosses the } \\ \text { dislocation line and } m \text { is to the left (right) } \\ \text { of } n \\ 0, \text { otherwise. }\end{array}\right.$

and $b$ is the lattice constant.
In terms of the static lattice Green's function $G_{i j}^{m n}=\left(\hat{\Phi}^{-1}\right)_{i j}^{m n}$, the displacement field, given by

$$
U_{i}^{m}=\sum_{n, j} G_{i j}^{m n} K_{j}^{n}
$$

with equation (2.1) for $K_{j}^{n}$ is shown in reference [2] to yield, far from the core, the well-known screw dislocation result of linear elastic theory.

Equations (2.1) and (2.2) are valid for pair-wise interactions over any distance in any lattice. Moreover, as discussed in reference 2 , the force equation (2.1) can be generalized in a straightforward manner to include anharmonic force terms. Thus, the discussion to follow, for a moving dislocation, is amenable to the same sort of generalization.

We shall extend equations $(2.1)$ to $(2.3)$ to the case where the screw dislocation travels down the $y$-axis, with its position at time $t$ given by $R(t) \hat{\mathbf{y}}$. For the case of a simple cubic lattice, the dependence of the function [r, $\left.\mathbf{r}^{\prime}\right]$ in (2.2) on the $x$-coordinates of $\mathbf{r}$ and $\mathbf{r}^{\prime}$ is described by $\theta\left(-r_{x}\right) \theta\left(r_{x}^{\prime}\right)-\theta\left(r_{x}\right) \theta\left(-r_{x}^{\prime}\right)$. Here $\theta(x)$ is the step-function, whose Fourier integral representation is

$$
\theta(x)=\frac{1}{2 \pi i} \int_{-\infty}^{\infty} \mathrm{d} k \frac{\mathrm{e}^{i x k}}{k-i \varepsilon}, \quad \varepsilon \rightarrow 0^{+}
$$

The dependence of $[\mathbf{r}, \mathbf{r}]$ on the $y$-coordinate, in the case of nearest-neighbour interactions - as considered from here onward - is given by $\theta\left(r_{y}-R(t)\right) \delta_{r_{y}, r_{y}^{\prime}}$ Then we can write

$$
K_{i}^{\mathrm{r}}(t)=b \delta_{i z} \sum_{\mathbf{r}^{\prime}}\left[\theta\left(-r_{x}\right) \theta\left(r_{x}^{\prime}\right)-\theta\left(r_{x}\right) \theta\left(-r_{x}^{\prime}\right)\right] \theta\left(r_{y}-R(t)\right) \Phi_{i 3}^{\mathrm{rr}}
$$

where the Kronecker $\delta^{\prime} \mathrm{s}, \delta_{r_{y}, r_{y}^{\prime}}$ are dropped, since the nearest-neighbour interactions will now be taken into account through the $\Phi^{\text {rr' }}$.

It is useful here to work in reciprocal space, where

$$
K_{i}(\mathbf{Q}, \omega) \equiv \frac{1}{\sqrt{2 \pi N}} \sum_{\mathbf{r}} \mathrm{e}^{-i \mathbf{Q} . \mathbf{r}} \int_{-\infty}^{\infty} \mathrm{d} t \mathrm{e}^{i \omega t} K_{i}^{\mathbf{r}}(t) .
$$

The sum on all $N$ discrete atomic positions $\mathbf{r}$ may be taken in the limit where $N \rightarrow \infty$, and hence $\mathbf{Q}$ may be considered continuous. We choose the origin such that the position of the atom labelled $(s, t, p)$ is

$$
\mathbf{r}=\left(r_{x}^{s}, r_{y}^{t}, r_{z}^{p}\right)=(s b+b / 2, t b, p b)
$$

for $s, t, p=0, \pm 1, \pm 2$.

Using equations (2.4) and (2.5) in (2.6), along with such useful identities as

$$
\frac{b}{2 \pi} \sum_{s=-\infty}^{\infty} \mathrm{e}^{i k r_{x}^{s}}=\sum_{h=-\infty}^{\infty}(-1)^{h} \delta\left(k-\frac{2 \pi}{b} h\right)
$$


we find after some straightforward computation that

$$
\begin{aligned}
K_{3}(\mathbf{Q}, \omega)=\frac{-\sqrt{2 \pi}}{2 b} \delta\left(Q_{z}\right)\left[\frac{\hat{\Phi}_{33}\left(Q_{x}, 0,0\right)-\hat{\Phi}_{33}(0,0,0)}{\sin b Q_{x} / 2}\right] \times \\
\times \sum_{h=-\infty}^{\infty} \frac{1}{Q_{y}+\frac{2 \pi}{b} h} \int_{-\infty}^{\infty} \mathrm{e}^{-i\left(Q_{y}+2 \pi h / b\right) R(t)+i \omega t} \mathrm{~d} t .
\end{aligned}
$$

Here, we have used the definition

$$
\hat{\boldsymbol{\Phi}}_{i j}(\mathbf{Q})=\frac{1}{\sqrt{N}} \sum_{\mathbf{r}} \Phi_{i j}^{\mathbf{r r} \mathbf{r}^{\prime}} \mathrm{e}^{-i \mathbf{Q}\left(\mathbf{r}-\mathbf{r}^{\prime}\right)}=\frac{1}{\sqrt{N}} \sum_{\mathbf{r}} \Phi_{i j}^{\mathbf{r} 0} \mathrm{e}^{i \mathbf{Q} \cdot \mathbf{r}},
$$

which for $i=j=3$, in the case of a simple-cubic lattice, with nearest-neighbour interactions, becomes

$$
\sqrt{N} \hat{\Phi}_{33}(\mathbf{Q})=2 \gamma_{L}\left[1-\cos b Q_{z}\right]+2 \gamma_{\mathrm{T}}\left[2-\cos b Q_{x}-\cos b Q_{y}\right]
$$

where $-\gamma_{\mathrm{T}}$ and $-\gamma_{\mathrm{L}}$ are the transverse and longitudinal force-constants.

Thus we finally have that $K_{x}(\mathbf{Q}, \omega)=K_{y}(\mathbf{Q}, \omega)=0$, and

$$
K_{3}(\mathbf{Q}, \omega)=-\frac{2 \sqrt{2 \pi}}{b \sqrt{N}} \gamma_{\mathrm{T}} \delta\left(Q_{z}\right) \sin \left(\frac{b Q_{x}}{2}\right) \times \sum_{h=-\infty}^{\infty} \frac{1}{Q_{y}+\frac{2 \pi}{b} h-i \varepsilon} \int_{-\infty}^{\infty} \mathrm{e}^{-i\left(Q_{y}+2 \pi h / b\right) R(t)+i \omega t} \mathrm{~d} t
$$

2 .1 The ATOMiC DisPlacements. - The general expression for the Fourier-time transform of the displacement of atom $m$ in the direction $\alpha$ is

$$
U_{\alpha}^{m}(\omega)=\frac{1}{\sqrt{N M}} \sum_{\mathbf{Q}, \sigma}^{\mathrm{BZ}} q(\mathbf{Q}, \sigma ; \omega) e_{\alpha}(\mathbf{Q}, \sigma) \mathrm{e}^{i \mathbf{Q} . \mathbf{r}^{m}}
$$

which can be expressed in terms of the phonon Green's function $G_{\alpha \beta}^{m n}(\omega)$ and the applied force $K_{\beta}^{n}(\omega)$ as

$$
U_{\alpha}^{m}(\omega)=\sum_{n} G_{\alpha \beta}^{m n}(\omega) K_{\beta}^{n}(\omega)
$$

where the summation in equation (2.13a) is over the Brillouin zone (BZ); $\mathbf{e}(\mathbf{Q}, \sigma)$ is the $\sigma$ th $(\sigma=1 \rightarrow 3)$ eigenvector of the dynamical matrix (and for a simple cubic lattice is given by $\left.e_{\alpha}(\mathbf{Q}, \sigma)=\delta_{\alpha, \sigma}\right) ;$ and $q(\mathbf{Q}, \sigma ; \omega)$ is the normal coordinate, which by inverting the above Fourier transform becomes :

$$
q(\mathbf{Q}, \sigma ; \omega)=\sqrt{N M} \sum_{\alpha, j} e_{\alpha}^{*}(\mathbf{Q}, \sigma) G_{\alpha j}(\mathbf{Q}, \omega) K_{j}(\mathbf{Q}, \omega)
$$

The Green's function in equations (2.13) and (2.14) refers to the perfect lattice (or else with a random array of point defects averaged over all configurations), whose translational symmetry permits one to use the definition

$$
G_{i j}(\mathbf{Q}, \omega)=\frac{1}{\sqrt{N}} \sum_{m} G_{i j}^{m n}(\omega) \mathrm{e}^{i \mathbf{Q} \cdot\left(\mathbf{r}^{m}-\mathbf{r}^{n}\right)}=\sum_{m} G_{i j}^{m 0}(\omega) \mathrm{e}^{i \mathbf{Q} \cdot \mathbf{r}^{m}}
$$

in obtaining (2.14). For the simple cubic lattice

$$
G_{i j}(\mathbf{Q}, \omega)=\frac{1}{\sqrt{N} M} \delta_{i j} \frac{1}{\omega^{2}(\mathbf{Q}, j)-\omega^{2}-i \varepsilon},
$$

in terms of the phonon frequency $\omega^{2}(\mathbf{Q}, j)$. On using equations (2.12) and $(2.15)$ in $(2.14)$, together with $M \omega^{2}(\mathbf{Q}, 3)=\sqrt{N} \hat{\Phi}_{33}(\mathbf{Q})$ as given by equation (2.11), we find that

$$
\begin{aligned}
q(\mathbf{Q}, \sigma ; \omega)=-\frac{2}{b}\left(\frac{2 \pi m}{N}\right)^{1 / 2} \gamma_{\mathrm{T}} \sin \left(\frac{b Q_{x}}{2}\right) & \delta\left(Q_{z}\right) \delta_{\sigma, 3} \times\left[2 \gamma_{\mathrm{T}}\left(2-\cos b Q_{x}-\cos b Q_{y}\right)-M \omega^{2}-i \varepsilon\right]^{-1} \times \\
& \times \sum_{h=-\infty}^{\infty}\left(Q_{y}+2 \pi h / b-i \varepsilon\right)^{-1} \int_{-\infty}^{\infty} \mathrm{d} t \mathrm{e}^{-i\left(Q_{y}+2 \pi h / b\right) R(t)+i \omega t}
\end{aligned}
$$


We can use this expression back in equation (2.13a) for the displacement, where we convert the sum over $\mathbf{Q}$ to an integral. Then

$$
\begin{aligned}
U_{\alpha}^{m}(t)= & \frac{1}{\sqrt{2 \pi}} \int_{-\infty}^{\infty} \mathrm{d} \omega \mathrm{e}^{-i \omega t} U_{\alpha}^{m}(\omega) \\
= & -\frac{b}{4 \pi^{3}} \delta_{\alpha, 3} \int_{-\infty}^{\infty} \mathrm{d} k_{y} \frac{\mathrm{e}^{i m_{y} k_{y}}}{k_{y}-i \varepsilon} \int_{-\infty}^{\infty} \mathrm{d} \omega \mathrm{e}^{-i \omega t} \times \\
& \quad \times \int_{-\pi}^{\pi} \mathrm{d} k_{x} \frac{\gamma_{\mathrm{T}} \sin \left(k_{x} / 2\right) \mathrm{e}^{i m_{x} k_{x}}}{4 \gamma_{\mathrm{T}}\left[\sin ^{2}\left(k_{x} / 2\right)+\sin ^{2}\left(k_{y} / 2\right)\right]-M \omega^{2}-i \varepsilon} \times \int_{-\infty}^{\infty} \mathrm{d} t^{\prime} \mathrm{e}^{-i k_{y} R\left(t^{\prime}\right) / b+i \omega t^{\prime}}
\end{aligned}
$$

where $k_{i}=Q_{i} b$ and $m_{i}=r_{i}^{m} / b$ for $i=x, y$. This expression is valid for a general $R(t)$. For the special case where $R(t)=v t$, the last integral in (2.17) becomes $2 \pi \delta\left(\omega-k_{y} v / b\right)$, permitting the immediate evaluation of the integral over $\omega$. The resulting equation is the equivalent of equation (2.9) in reference [9] by Celli and Flytzanis, thus confirming the validity of our equation (2.12) for the Fourier transformed, dynamical, Kanzaki force for a dislocation travelling with arbitrary time-displacement $R(t)$.

2.2 THE ENERGY DISSIPATION. - The power transferred to the lattice vibrations from an oscillating dislocation is given by the work performed by the Kanzaki force :

$$
\begin{aligned}
P(t) & =\sum_{m, i} K_{i}^{m}(t) \frac{\mathrm{d} u_{i}^{m}(t)}{\mathrm{d} t} \\
& =\frac{-i}{2 \pi} \sum_{m, i} \iint_{-\infty}^{\infty} \mathrm{d} \omega \mathrm{d} \omega^{\prime} \omega^{\prime} K_{i}^{m}(\omega) U_{i}^{m}\left(\omega^{\prime}\right) \mathrm{e}^{-i\left(\omega+\omega^{\prime}\right) t},
\end{aligned}
$$

where the Fourier-time-transform displacement is given by equation $(2.13 b)$ in terms of the phonon Green's function, with which we see

$$
P(\omega)=\frac{-i}{\sqrt{2 \pi}} \sum_{\substack{i, n \\ i, j}} \int_{-\infty}^{\infty} \mathrm{d} \omega^{\prime} \omega^{\prime} K_{i}^{m}\left(\omega-\omega^{\prime}\right) G_{i j}^{m n}\left(\omega^{\prime}\right) K_{j}^{n}\left(\omega^{\prime}\right),
$$

or

$$
P(\omega)=-i \sqrt{\frac{N}{2 \pi}} \sum_{i, j} \int_{-\infty}^{\infty} \mathrm{d} \omega^{\prime} \omega^{\prime} \sum_{\mathbf{Q}} K_{i}\left(\mathbf{Q}, \omega-\omega^{\prime}\right) G_{i j}(-\mathbf{Q}, \omega) K_{j}\left(-\mathbf{Q}, \omega^{\prime}\right)
$$

in terms of the Fourier-space-transforms defined in equations (2.6) and (2.15a).

For calculating the internal friction $Q^{-1}$, it is the work delivered per period of oscillation $T$ of the dislocation that must be determined.

$$
W=\int_{-T / 2}^{T / 2} P(t) \mathrm{d} t=\frac{2}{\sqrt{2 \pi}} \int_{-\infty}^{\infty} \mathrm{d} \omega \frac{\sin (\omega T / 2)}{\omega} P(\omega) .
$$

If $R(t)$ is an even function of time (e.g. $R(t)=R_{0} \cos \omega t$ ), then equation (2.5) shows that $K_{i}^{\mathrm{r}}(t)$ is also even in $t$, implying that $K_{i}(\mathbf{Q}, \omega)=K_{i}(\mathbf{Q},-\omega)$. Moreover, $G_{i j}^{m n}(\omega)=G_{i j}^{m n}(-\omega)^{*}$ implies (with inversion symmetry) that $G_{i j}(\mathbf{Q},+\omega)=G_{i j}(\mathbf{Q},-\omega)^{*}$. Then we can rewrite equations (2.19) as

$$
P(-\omega)=i \sqrt{\frac{N}{2 \pi}} \int_{-\infty}^{\infty} \mathrm{d} \omega^{\prime} \omega^{\prime} \sum_{\mathbf{Q}} K_{i}\left(\mathbf{Q}, \omega-\omega^{\prime}\right) G_{i j}\left(-\mathbf{Q}, \omega^{\prime}\right)^{*} K_{j}\left(-\mathbf{Q}, \omega^{\prime}\right),
$$

and with equations $(2.19 b)$ to $(2.21)$ we find

$$
\begin{aligned}
W & =\frac{2}{\sqrt{2 \pi}} \int_{0}^{\infty} \mathrm{d} \omega \frac{\sin (\omega T / 2)}{\omega}(P(\omega)+P(-\omega)) \\
& =\frac{2 \sqrt{N}}{\pi} \int_{-\infty}^{\infty} \mathrm{d} \omega^{\prime} \omega^{\prime} \sum_{\mathbf{Q}} \int_{0}^{\infty} \mathrm{d} \omega \frac{\sin (\omega T / 2)}{\omega} K_{i}\left(\mathbf{Q}, \omega-\omega^{\prime}\right) \operatorname{Im} G_{i j}\left(-\mathbf{Q}, \omega^{\prime}\right) K_{j}\left(-\mathbf{Q}, \omega^{\prime}\right) .
\end{aligned}
$$


Now substitute the Kanzaki force of (2.12) for the oscillatory screw dislocation in (2.22), replace the sum on $\mathbf{Q}$ by an integral, and integrate over $\omega$. Then after some manipulation, the energy dissipated becomes

$$
\begin{aligned}
W=\frac{16 \pi \gamma_{t}^{2}}{\sqrt{N} b^{2}}\left(\frac{L}{2 \pi}\right)^{4} & \int_{-\pi / b}^{\pi / b} \mathrm{~d} Q_{x} \int_{-\pi / b}^{\pi / b} \mathrm{~d} Q_{y} \sin ^{2}\left(\frac{b Q_{x}}{2}\right) \int_{0}^{\infty} \mathrm{d} \omega^{\prime} \cdot \omega^{\prime} \times \\
& \times \int_{-T / 2}^{T / 2} \mathrm{~d} t \mathrm{e}^{-i \omega^{\prime} t} \int_{-\infty}^{\infty} \mathrm{d} t^{\prime} \mathrm{e}^{i \omega^{\prime} t^{\prime}} \sum_{h, h^{\prime}=-\infty}^{\infty} \frac{\exp \left[-i\left(Q_{y}+\frac{2 \pi}{b} h\right) R(t)\right]}{Q_{y}+\frac{2 \pi}{b} h-i \varepsilon} \\
& \times \frac{\exp \left[i\left(Q_{y}+\frac{2 \pi}{b} h^{\prime}\right) R\left(t^{\prime}\right)\right]}{Q_{y}+\frac{2 \pi}{b} h^{\prime}+i \varepsilon} \operatorname{Im} G_{33}\left(-\mathbf{Q}_{\perp}, \omega^{\prime}\right),
\end{aligned}
$$

where $\mathbf{Q}_{\perp}=Q_{x} \hat{\mathbf{x}}+Q_{y} \hat{\mathbf{y}}$, and $L$ is the crystal length.

From equation $(2.15 b)$, for $\omega>0$ :

$$
\operatorname{Im} G_{33}(\mathbf{Q}, \omega)=\frac{\pi}{2 \sqrt{N} M} \frac{1}{\omega(\mathbf{Q}, 3)} \delta(\omega-\omega(\mathbf{Q}, 3))
$$

which used in (2.23) gives the general expression for the energy dissipated per cycle, in terms of the dislocation displacement function $R(t)$ :

$$
\begin{aligned}
& W=\frac{\gamma_{\mathrm{T}}^{2} b L}{2 \pi^{2} M} \iint_{-\pi / b}^{\pi / b} \mathrm{~d} Q_{x} \mathrm{~d} Q_{y} \sum_{h, h^{\prime}=-\infty}^{\infty} \frac{\sin ^{2}\left(b Q_{x} / 2\right)}{\left(Q_{y}-2 \pi h / b+i \varepsilon\right)\left(Q_{y}-2 \pi h^{\prime} / b-i \varepsilon\right)} \times \\
& \times \int_{-T / 2}^{T / 2} \mathrm{~d} t \mathrm{e}^{i\left(Q_{y}-2 \pi h / b\right) R(t)-i \omega\left(\mathbf{Q}_{\perp}, 3\right) t} \times \int_{-\infty}^{\infty} \mathrm{d} t^{\prime} \mathrm{e}^{-i\left(Q_{y}-2 \pi h^{\prime} b\right) R\left(t^{\prime}\right)+i \omega\left(\mathbf{Q}_{\perp}, 3\right) t^{\prime}} .
\end{aligned}
$$

\section{Energy dissipation from a harmonically oscillating dislocation.}

In this section we shall consider the energy dissipated by a harmonically vibrating screw dislocation, whose position along the $y$-axis is

$$
R(t)=b R_{0} \cos \Omega t,
$$

where $R_{0}$ is dimensionless (the amplitude measured in the number of lattice constants), as are the variables $\tau=\Omega t, k_{x}=b Q_{x}$, and $k_{y}=b Q_{y}$, which we now introduce in equation (2.25) to write

$$
\begin{aligned}
W=\frac{T^{2} \gamma_{t}^{2} b L}{8 \pi^{4} M} \int_{-\pi}^{\pi} \mathrm{d}^{2} k_{\perp} & \sum_{h, h^{\prime}=-\infty}^{\infty} \frac{\sin ^{2}\left(k_{x} / 2\right)}{\left(k_{y}-2 \pi h+i \varepsilon\right)\left(k_{y}-2 \pi h^{\prime}-i \varepsilon\right)} \times \\
\times & \sum_{m=0}^{\infty} \delta\left(m-\omega\left(\mathbf{k}_{\perp}, 3\right) / \Omega\right) \int_{-\pi}^{\pi} \mathrm{d} \tau \int_{-\pi}^{\pi} \mathrm{d} \tau^{\prime} \mathrm{e}^{i\left[\left(k_{y}-2 \pi h\right) R_{0} \cos \tau-\omega\left(\mathbf{k}_{\perp}, 3\right) \tau / \Omega\right]} \\
& \times \mathrm{e}^{-i\left[\left(k_{y}-2 \pi h^{\prime}\right) R_{0} \cos \tau^{\prime}-\omega\left(\mathbf{k}_{\perp}, 3\right) \tau^{\prime} / \Omega\right]^{\prime}}
\end{aligned}
$$

where $\mathbf{k}_{\perp}=k_{x} \hat{\mathbf{x}}+k_{y} \hat{\mathbf{y}}$. We see from (3.2) that there is a contribution to the energy emission from the oscillating dislocation whenever the frequency of a transverse phonon equals an integer times the (applied) frequency of the dislocation motion : $\omega\left(\mathbf{k}_{\perp}, 3\right)=m \Omega$. Now using the definition of the Bessel function

$$
i^{n} J_{n}(z)=\frac{1}{2 \pi} \int_{-\pi}^{\pi} \mathrm{e}^{-n i \theta-i z \cos \theta} \mathrm{d} \theta
$$

we find

$$
W=\frac{T^{2} \gamma_{t}^{2} b L}{2 \pi^{2} M} \sum_{m=0}^{\infty} \int_{-\pi}^{\pi} \mathrm{d}^{2} k_{\perp}\left|\sum_{h=-\infty}^{\infty} \frac{J_{m}\left(\left(k_{y}-2 \pi h\right) R_{0}\right)}{k_{y}-2 \pi h+i \varepsilon}\right|^{2} \times \sin ^{2}\left(k_{x} / 2\right) \delta\left(m-\omega\left(\mathbf{k}_{\perp}, 3\right) / \Omega\right) .
$$


For convenience in the calculations that follow, $R_{0}$ is chosen to be an odd integer divided by 2 , so that $R_{0}-1 / 2$ is an integer. In appendix $I$ it is shown that

$$
\sum_{h=-\infty}^{\infty} \frac{J_{m}\left(\left[k_{y}-2 \pi h\right] R_{0}\right)}{k_{y}-2 \pi h}=\frac{1}{\pi m} \times \begin{cases}2 \sum_{n=1}^{R_{0}-1 / 2} \sin \left(m \sin ^{-1} \frac{n}{R_{0}}\right) \sin \left(n k_{y}\right), & m=0,2,4, \ldots \\ 1+2 \sum_{n=1}^{R_{0}-1 / 2} \cos \left(m \sin ^{-1} \frac{n}{R_{0}}\right) \cos \left(n k_{y}\right), & m=1,3,5, \ldots\end{cases}
$$

With $M \omega^{2}\left(\mathbf{k}_{\perp}, 3\right)=\sqrt{N} \hat{\phi}_{33}\left(\mathbf{k}_{\perp} / b\right)$ and equation (2.11), and with the definition $q_{i}=k_{i} / 2$, the $\delta$-function in (3.4) becomes

$$
\delta(m-\omega(\mathbf{k}, 3) / \Omega)=\left\{\begin{array}{l}
\frac{M}{4 \gamma_{t}} m \Omega^{2}\left(\sin q_{x}^{0} \cos q_{y}^{0}\right)^{-1} \delta\left(q_{x}-q_{x}^{0}\right), \quad \text { if } q_{x}^{0} \text { as defined below, exists } \\
0, \text { otherwise }
\end{array}\right.
$$

Here $q_{x}^{0}$ as defined by

$$
q_{x}^{0}=\sin ^{-1}\left(\frac{M}{4 \gamma_{t}} m^{2} \Omega^{2}-\sin ^{2} q_{y}\right)^{1 / 2}
$$

exists as a real zero of the argument of the $\delta$-function only if

$$
\alpha^{2} m^{2}-1 \leqslant \sin ^{2} q_{y} \leqslant \alpha^{2} m^{2}
$$

where

$$
\alpha^{2} \equiv \frac{M \Omega^{2}}{4 \gamma_{t}}=\frac{\Omega^{2}}{\omega^{2}\left(\frac{\pi}{b} \hat{\mathbf{x}}, 3\right)}
$$

The condition on $q_{y}$ and $m$ given by (3.8), and hereafter called condition 1 , can be written equivalently as :

$$
\omega\left(Q_{y} \hat{\mathbf{y}}, 3\right) \leqslant m \Omega \leqslant \omega\left(\frac{\pi}{b} \hat{\mathbf{x}}+Q_{y} \hat{\mathbf{y}}, 3\right)
$$

which simply means that the integer multiple $m$ of the applied frequency must lie within the range of transverse phonon frequencies for $Q_{z}=0$ at a given $Q_{y}$.

Equation (3.4), together with (3.5) to (3.9), then becomes

$$
W=\frac{8 \gamma_{\mathrm{T}} b L}{\pi^{2}} \underbrace{\sum_{m=0}^{\infty} \int_{0}^{\pi / 2}}_{\text {cond. } 1} \mathrm{~d} q_{y} \frac{1}{m}\left(\frac{\alpha^{2} m^{2}-\sin ^{2} q_{y}}{1-\alpha^{2} m^{2}+\sin ^{2} q_{y}}\right)^{1 / 2} \times \begin{cases}E^{2}\left(m, q_{y}\right) ; & m=0,2,4, \ldots \\ D^{2}\left(m, q_{y}\right) ; & m=1,3,5, \ldots\end{cases}
$$

where

$$
\begin{aligned}
& E\left(m, q_{y}\right)=2 \sum_{n=1}^{R_{0}-1 / 2} \sin \left(m \sin ^{-1} \frac{n}{R_{0}}\right) \sin \left(2 n q_{y}\right) \\
& D\left(m, q_{y}\right)=1+2 \sum_{n=1}^{R_{0}-1 / 2} \cos \left(m \sin ^{-1} \frac{n}{R_{0}}\right) \cos \left(2 n q_{y}\right) .
\end{aligned}
$$

In order to evaluate equation (3.11), it is necessary to consider more carefully the condition 1 on $m$ and $q_{y}$, as expressed in equation (3.8). First, look at values of $m$ such that $0 \leqslant m \leqslant 1 / \alpha$, which implies that $0 \leqslant \alpha^{2} m^{2} \leqslant 1$ and $-1 \leqslant \alpha^{2} m^{2}-1 \leqslant 0$. Condition 1 then requires that the integration on $q_{y}$ extend from $q_{y}=0$ to $q_{y}=\sin ^{-1} \alpha m$. Second, for values of $m$ such that $1 / \alpha<m<\sqrt{2} / \alpha$, note that $1 \leqslant \alpha^{2} m^{2}<2$ and $0<\alpha^{2} m^{2}-1<1$, so that condition 1 requires integration from $q_{y}=\sin ^{-1}\left(\alpha^{2} m^{2}-1\right)^{1 / 2}$ to $\pi / 2$. Finally, values of $m$ such that $\sqrt{2} / \alpha<m$, implying that $2<\alpha^{2} m^{2}$ and $1<\alpha^{2} m^{2}-1$, are excluded by condition 1 . 
Hence,

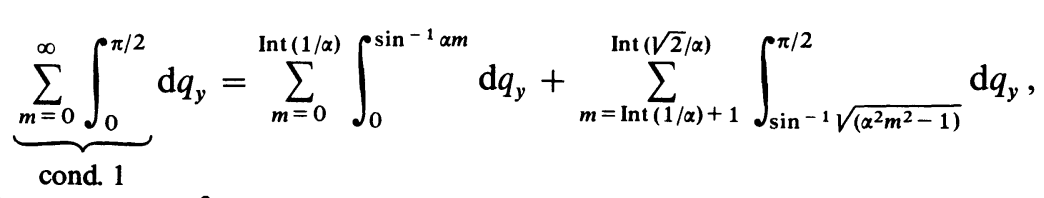

where Int $(x)$ is the integer part of $x$.

With the substitution $x=\sin q_{y}$ and $y=x / \alpha m$ and with the separation of terms in even and odd $m(1 / \alpha$ is taken as an even integer for convenience), the energy transferred to the lattice vibrations is given by

$$
\begin{aligned}
& \left(\frac{\pi^{2}}{8 \gamma_{t} b L}\right) W=\sum_{m=1}^{1 / 2 \alpha} \alpha\left\{\int_{0}^{1} \mathrm{~d} y\left[\frac{1-y^{2}}{(2 m \alpha)^{-2}-\left(1-y^{2}\right)}\right]^{1 / 2}\left[\frac{1}{1-(2 m \alpha y)^{2}}\right]^{1 / 2} E^{2}\left(2 m, \sin ^{-1} 2 m \alpha y\right)+\right. \\
& \left.\quad+\int_{0}^{1} \mathrm{~d} y\left[\frac{1-y^{2}}{([2 m-1] \alpha)^{-2}-\left(1-y^{2}\right)}\right]^{1 / 2}\left[\frac{1}{1-([2 m-1] \alpha y)^{2}}\right]^{1 / 2} D^{2}\left(2 m-1, \sin ^{-1}(2 m-1) \alpha y\right)\right\} \\
& \quad+\sum_{m=\frac{1}{2 \alpha}+1}^{\ln (\sqrt{2} / 2 \alpha)}\left\{\frac{1}{2 m} \int_{f_{m}}^{1} \mathrm{~d} x\left[\frac{(2 m \alpha)^{2}-x^{2}}{1-(2 m \alpha)^{2}+x^{2}}\right]^{1 / 2}\left[\frac{1}{1-x^{2}}\right]^{1 / 2} E^{2}\left(2 m, \sin ^{-1} x\right)\right. \\
& \left.\quad+\frac{1}{2 m-1} \int_{g_{m}}^{1} \mathrm{~d} x\left[\frac{(2 m-1)^{2} \alpha^{2}-x^{2}}{1-(2 m-1)^{2} \alpha^{2}+x^{2}}\right]^{1 / 2}\left[\frac{1}{1-x^{2}}\right]^{1 / 2} D^{2}\left(2 m-1, \sin ^{-1} x\right)\right\}
\end{aligned}
$$

where $f_{m} \equiv\left[(2 m \alpha)^{2}-1\right]^{1 / 2}$ and $g_{m} \equiv\left[(2 m-1)^{2} \alpha^{2}-1\right]^{1 / 2}$.

Up to this point no approximations have been made in the calculations, and thus the result in (3.14) is exact, for all frequencies of oscillation. From here onward we shall assume that the dislocation oscillation frequency $\Omega \ll \omega\left(\frac{\pi}{b} \hat{\mathbf{x}}, 3\right)$, so that $\alpha \ll 1$ (e.g. if we assume $\Omega \leqslant 10^{7} \mathrm{~Hz}$ and $\omega\left(\frac{\pi}{b} \hat{\mathbf{x}}, 3\right) \approx 10^{12} \mathrm{~Hz}$, then $\alpha \leqslant 10^{-5}$ ).

For $\alpha \ll 1$, take $z=\alpha m$ as a continuous variable, and convert the summations on $m$ to integrals :

$$
\begin{aligned}
\left(\frac{\pi^{2}}{8 \gamma_{\mathrm{t}} b L}\right) W=\int_{0}^{1 / 2} \mathrm{~d} z \int_{0}^{1} \mathrm{~d} y & 2 z\left(\frac{1-y^{2}}{1-4 z^{2}\left(1-y^{2}\right)}\right)^{1 / 2}\left(\frac{1}{1-4 z^{2} y^{2}}\right)^{1 / 2} F\left(\frac{2 z}{\alpha}, \sin ^{-1} 2 z y\right)+ \\
& +\int_{1 / 2}^{\sqrt{2} / 2} \frac{\mathrm{d} z}{2 z} \int_{\sqrt{4 z^{2}-1}}^{1} \mathrm{~d} x\left(\frac{4 z^{2}-x^{2}}{1-4 z^{2}+x^{2}}\right)^{1 / 2}\left(\frac{1}{1-x^{2}}\right)^{1 / 2} F\left(\frac{2 z}{\alpha}, \sin ^{-1} x\right)
\end{aligned}
$$

where

$$
\begin{aligned}
F\left(\frac{2 z}{\alpha}, \beta\right) \equiv E^{2}\left(\frac{2 z}{\alpha}, \beta\right) & +D^{2}\left(\frac{2 z}{\alpha}, \beta\right)=1+2 R_{0}+ \\
& +2 \sum_{n=1}^{R_{0}-1 / 2}\left[2 \cos \left(\frac{2 z}{\alpha} \sin ^{-1} \frac{n}{R_{0}}\right) \cos (2 n \beta)+\cos \left(\frac{4 z}{\alpha} \sin ^{-1} \frac{n}{R_{0}}\right) \cos (4 n \beta)\right] \\
& +2 \sum_{\substack{n, n^{\prime}=1 \\
n \neq n^{\prime}}}^{R_{0}-1 / 2}\left[\cos \left(\frac{2 z}{\alpha}\left[\sin ^{-1} \frac{n}{R_{0}}-\sin ^{-1} \frac{n^{\prime}}{R_{0}}\right]\right) \cos \left(2\left[n-n^{\prime}\right] \beta\right)\right. \\
& \left.+\cos \left(\frac{2 z}{\alpha}\left[\sin ^{-1} \frac{n}{R_{0}}+\sin ^{-1} \frac{n^{\prime}}{R_{0}}\right]\right) \cos \left(2\left[n+n^{\prime}\right] \beta\right)\right] .
\end{aligned}
$$

Equation (3.15) now gives us an expression for the energy dissipated per cycle - an expression valid for all oscillation frequencies well under $\mathrm{THz}$ - which requires only the evaluation of some finite sums and finite integrals. The integral over $1+2 R_{0}$ can be carried out directly, while the integrals over the terms $\cos z \frac{\gamma}{\alpha}$ (where $\gamma=2$ or $4 \times \sin ^{-1} \frac{n}{R_{0}}$ or else $\gamma=2 \sin ^{-1} \frac{n}{R_{0}} \pm 2 \sin ^{-1} \frac{n^{\prime}}{R_{0}}$, with $n \neq n^{\prime}$ ) are of a form amenable to asymptotic expansion in $\alpha / \gamma$.

To see this, first note that $\alpha / \gamma$ is small for reasonable choice of the physical parameters : the smallest value of $\gamma$ is $2 \sin ^{-1} \frac{1}{R_{0}}$, which for $R_{0} \gg 1$ is $\approx \frac{2}{R_{0}}$; and thus for $R_{0}<200$ and $\Omega \leqslant 10^{6} \mathrm{~s}^{-1}$, $\alpha / \gamma \lesssim \Omega R_{0} / 2 \omega(\hat{\mathbf{x}} \pi / b, 3) \leqslant 10^{-4}$. Next, note that the integrands are properly integrable. The first integral in 
equation (3.15) is made up of terms like

$$
\int_{-1 / 2}^{1 / 2} \mathrm{~d} z\left[|z| \int_{0}^{1} \mathrm{~d} y\left(\frac{1-y^{2}}{1-4 z^{2}\left(1-y^{2}\right)} \cdot \frac{1}{1-4 z^{2} y^{2}}\right)^{1 / 2} \cos \left(2 n \sin ^{-1} 2 z y\right)\right] \cos z \frac{\gamma}{\alpha},
$$

where the integrand of the $z$-integration diverges only logarithmically at $z= \pm 1 / 2$, and thus is integrable on $[-1 / 2,1 / 2]$. The second integral in equation (3.15) is made up of terms like

$$
\int_{1 / 2}^{\sqrt{2} / 2} \mathrm{~d} z\left[\frac{1}{z} \int_{\sqrt{4 z^{2}-1}}^{1} \mathrm{~d} x\left(\frac{4 z^{2}-x^{2}}{1-4 z^{2}+x^{2}} \cdot \frac{1}{1-x^{2}}\right)^{1 / 2} \cos \left(2 n \sin ^{-1} x\right)\right] \cos z \frac{\gamma}{\alpha}
$$

which can be put into the form :

$$
1 / 2 \cos \frac{\sqrt{2} \gamma}{2 \alpha} \int_{-(\sqrt{2-1})}^{(\sqrt{2-1)}} \mathrm{d} z g_{\mathrm{c}}(z) \cos \frac{\gamma}{2 \alpha} z-1 / 2 \sin \frac{\sqrt{2} \gamma}{2 \alpha} \int_{-(\sqrt{2-1})}^{(\sqrt{2-1})} \mathrm{d} z g_{\mathrm{s}}(z) \sin \frac{\gamma}{2 \alpha} z
$$

where for $-(\sqrt{2}-1) \leqslant z \leqslant 0$,

$$
g_{\mathrm{c}}(z)=g_{\mathrm{s}}(z)=g(z) \equiv \frac{1}{z+\sqrt{2}} \int_{\left[(z+\sqrt{2})^{2}-1\right]^{1 / 2}}^{1} \mathrm{~d} x\left[\frac{(z+\sqrt{2})^{2}-x^{2}}{1-(z+\sqrt{2})^{2}+x^{2}} \cdot \frac{1}{1-x^{2}}\right]^{1 / 2} \cos \left(2 n \sin ^{-1} x\right)
$$

and for $0 \leqslant z \leqslant(\sqrt{2}-1), g_{\mathrm{c}}(z)=-g_{\mathrm{s}}(z)=g(-z)$. Again, the integrands of the $z$-integrals contain divergences, but only logarithmic ones at the end-points, and are thus integrable over $[-(\sqrt{2}-1),+(\sqrt{2}-1)]$.

The Riemann-Lebesgue Lemma states that if $f(x)$ is integrable on $[-\pi, \pi]$, then

$$
\operatorname{Lim}_{t \rightarrow \infty} \int_{-\pi}^{\pi} \mathrm{d} x f(x) \cos t x=0
$$

and furthermore, if $f(x)$ is a polynomial the integral approaches zero as $1 / t$ [13].

In the present work, equation (3.15) is evaluated to lowest order, i.e., only the $1+2 R_{0}$ term from (3.16) is used, and the terms which are of the order $\alpha / \gamma \approx 10^{-4}$ or smaller (containing the $\Omega$-dependence) are left for future work. Thus to zeroth order in $\alpha / \gamma$ we have

$$
\begin{aligned}
\left(\frac{\pi^{2}}{8 \gamma_{\mathrm{t}} b L}\right) W^{(0)}=\left(1+2 R_{0}\right)\left[2 \int_{0}^{1 / 2} \mathrm{~d} z z \int_{0}^{1} \mathrm{~d} y\left(\frac{1-y^{2}}{1-4 z^{2}\left(1-y^{2}\right)} \cdot \frac{1}{1-4 z^{2} y^{2}}\right)^{1 / 2}+\right. & \\
& \left.+\frac{1}{2} \int_{1 / 2}^{\sqrt{2} / 2} \mathrm{~d} z \frac{1}{z} \int_{\sqrt{4 z^{2}-1}}^{1} \mathrm{~d} x\left(\frac{4 z^{2}-x^{2}}{1-4 z^{2}+x^{2}} \cdot \frac{1}{1-x^{2}}\right)^{1 / 2}\right]
\end{aligned}
$$

which becomes (see, for example, Ref. [13]) :

$$
\begin{aligned}
& W^{(0)}=\frac{8 \gamma_{t} b}{\pi^{2}} L\left(1+2 R_{0}\right)\left\{\frac{1}{4} \int_{0}^{1} \mathrm{~d} y \frac{1}{y} \ln \left(2 y\left(1-y^{2}\right)^{1 / 2}+1\right)+\right. \\
& \left.\quad+\frac{1}{2} \int_{0}^{1} \mathrm{~d} x x\left[K\left(x \sqrt{2-x^{2}}\right)-\frac{1-x^{2}}{2-x^{2}} \Pi\left(\frac{\pi}{2}, x^{2}, x \sqrt{2-x^{2}}\right)\right]\right\},
\end{aligned}
$$

where

$$
K(k)=\int_{0}^{\pi / 2} \frac{\mathrm{d} \theta}{\left(1-k^{2} \sin ^{2} \theta\right)^{1 / 2}}
$$

is the complete elliptic integral of the first kind, and

$$
\Pi\left(\frac{\pi}{2}, n, k\right)=\int_{0}^{\pi / 2} \frac{\mathrm{d} \theta}{\left(1+n \sin ^{2} \theta\right)\left(1-k^{2} \sin ^{2} \theta\right)^{1 / 2}}
$$

is the complete elliptic integral of the third kind. With numerical integration of the integrals in (3.18), it is found 
that

$$
W^{(0)}=\gamma_{\mathrm{t}} b L\left(1+2 R_{0}\right)(0.638) .
$$

Remember that $L$ being the crystal dimension is also the dislocation length; and in any experiment in which the crystal is subjected to a real applied strain $1+2 R_{0} \approx 2 R_{0}$, and so $W^{(0)}$ is proportional to $2 R_{0} L / b$, the number of atomic bonds broken by the dislocation, times the harmonic bond-energy $\gamma_{\mathrm{t}} b^{2}$.

In other words, the energy given by the applied external force to each core atom, in order to push it up to the top of the hill in the interatomic potential, is largely radiated away into the crystal as that atom comes swiftly down the other side of the hill into the next valley. The time scale for the atom to relax into this new valley - on the order of an inverse phonon frequency $10^{-12} \mathrm{~s}$ - is much shorter than the oscillation period of the applied force, thus allowing the atom to decouple for a brief time (time $=$ well-width divided by the average velocity of the dislocation) from the applied force, long enough to lose its elastic energy. In the present snappingbond model, the interatomic potential wells have sharp cusps between them, but smoothing out these cusps will not change the basic picture (as long as the well-width divided by the average dislocation velocity remains large compared to the atomic relaxation time - which depends on the slope of the well-wall).

That equation (3.21) is intuitively the correct result serves to confirm the validity of (3.15), whose evaluation to the next order in $\alpha / \gamma$ would provide the frequency-dependent dissipation (dynamic Peierls energy).

\section{Possible extensions of the model}

The advantage of the formalism developed here is the unambiguous way in which it can be extended and generalized : the effects of a finite temperature or of a random array of point defects can be incorporated into equation (2.23) through the function, $\operatorname{Im} G$. The simplest approach is to replace the $\delta$-function, in $(2.24)$ by a Lorentzian with a width that is dependent on temperature or on defect-concentration.

The configuration-averaged Green's function for a random array of point defects, of concentration $c$, gives in the average- $t$-matrix approximation (for example, see Ref. [14])

$$
\operatorname{Im}\left\langle G_{33}(\mathbf{Q} \omega)\right\rangle=\frac{-\Sigma_{2}(\mathbf{Q} \omega)}{\left[M\left(\omega^{2}(\mathbf{Q} 3)-\omega^{2}\right)+\Sigma_{1}(\mathbf{Q} \omega)\right]^{2}+\left[\Sigma_{2}(\mathbf{Q} \omega)\right]^{2}}
$$

where for a defect of mass-change $\Delta M=M-M_{\text {def }}$, the imaginary and real parts of the self-energy are

$$
\begin{aligned}
& \Sigma_{2}=-C \frac{\stackrel{\circ}{G}^{\prime \prime}(\omega)}{\left[\frac{1-\stackrel{\circ}{G}^{\prime}(\omega) \Delta M \omega^{2}}{\Delta M \omega^{2}}\right]^{2}+\stackrel{\circ}{G}^{\prime \prime}(\omega)^{2}} \\
& \Sigma_{1}=-C \frac{\Delta M \omega^{2}-\left(1-\stackrel{\circ}{\left.G^{\prime} \Delta M \omega^{2}\right)}\right.}{\left(1-\stackrel{\circ}{G}^{\prime} \Delta M \omega^{2}\right)^{2}+\left(\stackrel{\circ}{G}^{\prime \prime} \Delta M \omega^{2}\right)^{2}} .
\end{aligned}
$$

Here $\stackrel{\circ}{G}$ is the perfect lattice Green's function : $\stackrel{\circ}{G z}_{z z}(\omega)=\stackrel{\circ}{G}^{\prime}(\omega)+i \stackrel{\circ}{G}^{\prime \prime}(\omega)$. In the low frequency (Debye) approximation :

$$
\begin{gathered}
\stackrel{\circ}{G}^{\prime}=\frac{3}{M \omega_{\mathrm{D}}^{3}}\left\{1-\frac{\omega}{\omega_{\mathrm{D}}} \tan ^{-1} \frac{\omega}{\omega_{\mathrm{D}}}\right\} \\
\stackrel{\circ}{G^{\prime \prime}}=\frac{3 \pi}{2 M \omega_{\mathrm{D}}^{3}} \omega,
\end{gathered}
$$

where $\omega_{\mathrm{D}}$ is the Debye frequency.

It is immediately obvious that $\omega \operatorname{Im}\langle G(\omega)\rangle$ is an even function of $\omega$, so that the frequency integration in equation (2.23) can be rewritten as

$$
I=\frac{1}{2} \int_{-\infty}^{\infty} \mathrm{d} \omega^{\prime} \omega^{\prime} \operatorname{Im}\left\langle G\left(\omega^{\prime}\right)\right\rangle \mathrm{e}^{-i\left(t-t^{\prime}\right) \omega^{\prime}}
$$

Since the width $\Sigma_{2}$ of the function $\operatorname{Im}\langle G(\omega)\rangle$ is proportional to $c$, then for $c \ll 1$, the width is very narrow; and thus both $\Sigma_{1}(\omega)$ and $\Sigma_{2}(\omega)$ may be evaluated at the peak-centre frequency of $\operatorname{Im}\langle G(\omega)\rangle$, namely, 
$\omega=\omega(\mathbf{Q} 3) \equiv \stackrel{\circ}{\omega}$. The frequency integral becomes :

$$
\begin{aligned}
I & =\frac{\stackrel{\circ}{\omega} \Sigma_{2}(\stackrel{\circ}{\omega})}{2 M^{2} \sqrt{N}} \int_{-\infty}^{\infty} \mathrm{d} \omega \frac{\mathrm{e}^{-i\left(t-t^{\prime}\right) \omega}}{\left[\stackrel{\circ}{\omega}^{2}+\Sigma_{1}(\stackrel{\circ}{\omega})^{2} / M-\omega^{2}\right]^{2}+\left[\frac{\Sigma_{2}(\stackrel{\circ}{\omega})}{M}\right]^{2}} \\
& =\frac{\pi}{4} \frac{\stackrel{\circ}{\omega}}{M \sqrt{N}}\left\{\frac{\mathrm{e}^{i\left|t-t^{\prime}\right| \cdot\left[\stackrel{\circ}{\omega}^{2}+\Sigma_{1}^{2} / M+i\left|\Sigma_{2}\right| / M\right]^{1 / 2}}}{\left[\circ^{2}+\Sigma_{1}^{2} / M+i\left|\Sigma_{2}\right| / M\right]^{1 / 2}}+c .\right\} .
\end{aligned}
$$

Again under the assumption that $\Sigma_{2}$ is small, we can expand the square-roots to obtain :

$$
I=\frac{\pi}{2} \frac{\stackrel{\circ}{\omega}}{M \sqrt{N}} \operatorname{Re}\left\{\left(\frac{1}{\bar{\omega}}+i \frac{c \gamma}{\bar{\omega}^{3}}\right) \mathrm{e}^{-i\left|t-t^{\prime}\right| \bar{\omega}}\right\} \mathrm{e}^{-\left|t-t^{\prime}\right| c \gamma / \bar{\omega}},
$$

where $\bar{\omega} \equiv\left(\stackrel{\circ}{\omega}^{2}+\Sigma_{1}^{2} / M\right)^{1 / 2}$ and $\gamma=\frac{\left|\Sigma_{2}\right|}{2 c M}$. Equation (4.6) can be used in (2.23) for the $\omega$-integration. It is obvious from (4.6) that this reduces to the previous result in the limit where $c \rightarrow 0$. A full evaluation of the integrals for the point-defect problem is beyond the scope of this work. Nevertheless, the form of equation (4.6) allows us to see that the change in the energy dissipation due to the defects is proportional to $c$ in its leading order - as postulated in the phenomenological model of the peaking-effect in reference [6]. Furthermore, note that a low frequency resonance created by a force-constant instability, $f^{\text {eff. }} \rightarrow 0$, as is associated with the dumbbell interstitial (see Ref. [14]) could lead to a very large resonant-enhancement of $\Sigma_{2}$ and of the energy dissipation. This may play a role in the peaking-effect and shall be explored.

The simplest calculation, within this formalism, to see the effects of point defects on the lattice dynamics of a moving dislocation, would be the one for a constant dislocation velocity and a random array of isotopic mass defects : substitute $R(t)=v t$ into (2.23) and then use (4.6). Such a calculation is currently being pursued.

\section{Discussion.}

Even allowing for the possible extensions mentioned in the previous section, e.g., anharmonicity in the core and phonon damping at finite temperature, the basic model of dislocation dynamics presented here would remain an idealized one, in that the dislocation line moves rigidly; there are no kinks or jogs or dissociation into partial dislocations. Given the great mathematical difficulties inherent in even an idealized lattice model, it is obvious that a complete atomistic description of dislocation dynamics, which is all inclusive with respect to the complex reality of crystalline imperfections and mechanical behaviour, is still beyond reach. Nonetheless, the importance of processes that occur on an atomic scale (e.g., the interaction between point defects and dislocations) dictates that a lattice dynamical model, even an idealized one, be created.

Indeed, the lattice dynamical model presented here, idealized though it may be, yields results that should be of physical significance - results not found in classical elasticity theories. Granted that the elastic energy associated with the dislocation-bowing provides the restoring force to enable it to oscillate, one can simply assume a long vibrating dislocation, as a given, and then treat sections of it as straight lines. In that case, the results here indicate that through the mechanism of bond breaking (after Celli), there is an additional source of dislocation damping - a source which is present at zero temperature and frequency. This source of energy dissipation might, perhaps, help to explain the anomalously high internal friction measured at submegahertz frequencies and at low temperatures. Moreover, the dependence of the energy dissipation upon the bulk concentration of point defects indicates that there exists a global influence, via the phonons, of the defects upon the dislocation damping. This influence, whose magnitude has not yet been calculated, would be in addition to any shorter range dislocation-defect interactions that depend on the number of defects reaching the dislocation line. Such a global influence is in fact indicated in the pulsed irradiation experiments of Caro and Mondino on the " peaking-effect " (see Ref. [3]).

A source of dislocation energy dissipation as indicated here - namely, one independent of dislocation velocity but dependent on the number of bonds broken (i.e. on the area swept by the dislocation), with an added term proportional to the bulk volume - density of point defects - has recently been employed in a phenomenological model (see Ref. [6]) of the " peaking effect ». That model also included a short range interaction between the dislocation and point defects (acting as pinning centres on the dislocation line) and a strain dependent depinning mechanism. The model was successfully fit to all the experimentally observed features of the " peaking effect ». Thus, experiment lends credence to the lattice dynamical model of dislocation motion proposed here. 


\section{Acknowledgments.}

The greater part of this work was carried out while the author was employed by the IGA-EPFL, and was supported by the Swiss National Science Foundation through Grant No. 2.175 078, which is gratefully acknowledged. Special thanks to Prof. W. Benoit.

Thanks also to Professor P. H. Dederichs for his suggestions and to Professor A. A. Maradudin for reading the manuscript.

\section{Appendix I.}

To evaluate

$$
S \equiv \sum_{l=-\infty}^{\infty} \frac{J_{m}([Q-2 \pi l] R)}{Q-2 \pi l}
$$

we begin with the recurrence relation to write

$$
S=\sum_{l=-\infty}^{\infty} \frac{R}{2 m}\left\{J_{m-1}([Q-2 \pi l] R)+J_{m+1}([Q-2 \pi l] R)\right\}
$$

and then from reference [15] (Eq. (57.17.1)),

$$
\begin{aligned}
S & =\frac{R}{2 m} \sum_{l=-\infty}^{\infty}\left\{\sum_{s=-\infty}^{\infty} J_{s}(Q R) J_{m-1-s}(-2 \pi l R)+\sum_{s=-\infty}^{\infty} J_{s}(Q R) J_{m+1-s}(-2 \pi l R)\right\} \\
& =\frac{R}{2 m} \sum_{s=-\infty}^{\infty} J_{s}(Q R)(-1)^{m-s-1} \sum_{l=-\infty}^{\infty}\left\{J_{m-s-1}(2 \pi R l)+J_{m-s+1}(2 \pi R l)\right\} .
\end{aligned}
$$

If $m-s \pm 1$ is odd, the summation on $l$ gives zero, and thus we set $m-s-1=2 n$ and sum on $n$ :

$$
S=\frac{R}{2 m} \sum_{n=-\infty}^{\infty}\left[J_{m-1-2 n}(Q R)+J_{m+1-2 n}(Q R)\right]\left[J_{2 n}(0)+2 \sum_{l=1}^{\infty} J_{2 n}(2 \pi R l)\right] .
$$

From reference [15] (Eq. (57.27.1)),

$$
\sum_{l=1}^{\infty} J_{2 n}(2 \pi R l)=\frac{1}{2 \pi R}-\frac{1}{2} \delta_{n, 0}+2 \sum_{k=1}^{R-1 / 2}\left([2 \pi R]^{2}-4 \pi^{2} k^{2}\right)^{-1 / 2} \cos \left(2 n \sin ^{-1} \frac{k}{R}\right),
$$

which implies that

$$
\begin{aligned}
S=\frac{1}{2 \pi m} \sum_{n=-\infty}^{\infty}\left[J_{m-1-2 n}(Q R)+J_{m+1-2 n}(Q R)\right]+ & \\
& +\frac{1}{\pi m} \sum_{k=1}^{R-1 / 2}\left(1-\frac{k^{2}}{R^{2}}\right)^{-1 / 2} \sum_{n=-\infty}^{\infty}\left[J_{m-1-2 n}(Q R)+J_{m+1-2 n}(Q R)\right] \cos \left(2 n \sin ^{-1} \frac{k}{R}\right)
\end{aligned}
$$

which together with reference [15] (Eq. (57.1.13)) gives

$$
\begin{aligned}
S=\frac{1}{2 \pi m} \times\left\{\begin{array}{l}
2 ; m=1,3,5, \ldots \\
0 ; m=0,2,4
\end{array}\right. \\
+\frac{1}{\pi m} \sum_{k=1}^{R-1 / 2}\left(1-\frac{k^{2}}{R^{2}}\right)^{-1 / 2} \sum_{n=-\infty}^{\infty}\left[J_{m-1-2 n}(Q R)+J_{m+1-2 n}(Q R)\right] \cos \left(2 n \sin ^{-1} \frac{\mathrm{k}}{R}\right) .
\end{aligned}
$$

From reference [15] (Eq. (74.2.22)) we have that

$$
\sum_{l=0}^{\infty} J_{2 l}(Q R) \cos 2 l y=\frac{1}{2} \cos (Q R \sin y)+\frac{1}{2} J_{0}(Q R),
$$

with which we can show that for $m \pm 1=0,2,4, \ldots$

$$
\sum_{n=-\infty}^{\infty} J_{m \pm 1-2 n}(Q R) \cos (2 n y)=\cos ([m \pm 1] y) \cos (Q R \sin y) .
$$


Similarly, from reference [15] (Eq. (74.2.26)) we have that

$$
\sum_{l=0}^{\infty} J_{2 l+1}(Q R) \sin (2 l+1) y=\frac{1}{2} \sin (Q R \sin y),
$$

with which we can show that for $m \pm 1=-1,1,3, \ldots$

$$
\sum_{n=-\infty}^{\infty} J_{m \pm 1-2 n}(Q R) \cos (2 n y)=\sin ([m \pm 1] y) \sin (Q R \sin y) .
$$

Now, using equation (A.10) and (A.12) with $y=\sin ^{-1} \frac{k}{R}$ in (A.8), we find :

$$
S=\frac{1}{\pi m} \times\left\{\begin{array}{lll}
2 \sum_{k=1}^{R-1 / 2} \sin \left(m \sin ^{-1} \frac{k}{R}\right) \sin (Q k) ; & \text { if } & m=0,2,4, \ldots \\
1+2 \sum_{k=1}^{R-1 / 2} \cos \left(m \sin ^{-1} \frac{k}{R}\right) \cos (Q k) ; & \text { if } & m=1,3,5, \ldots
\end{array}\right.
$$

\section{References}

[1] Maradudin, A. A., J. Phys. Chem. Solids 9 (1958) 1.

[2] Boyer, L. L. and HARdy, J. R.,, Philos. Mag. 24 (1971) 647.

[3] Caro, J. A. and Mondino, M., J. Appl. Phys. 52 (1981) 7147.

[4] Simpson, H. M., Sosin, A. and Seiffert, L., J. Appl. Phys. 42 (1971) 3977.

[5] Simpson, H. M., Sosin, A., Edwards, G. R. and SeIFFert, S. L., Phys. Rev. Lett. 26 (1971) 897.

[6] Caro, J. A., Glass, N. E. and Mondino, M. A., J. Appl. Phys. 53 (1982) 4854.

[7] Ishioka, S., J. Phys. Soc. Japan 34 (1973) 462.

[8] Atkinson, W. and Cabrera, N., Phys. Rev. A 138 (1965) 763.

[9] Celli, V. and Flytzanis, N., J. Appl. Phys. 41 (1970) 4443.
[10] Flytzanis, N. and Celli, V., J. Appl. Phys. 43 (1972) 3301.

[11] Celli, V., Flytzanis, N. and Crowley, S., J. Phys. Chem. Solids 37 (1976) 1125.

[12] Flytzanis, N., Crowley, S., Celli, V., J. Phys. Chem. Solids 38 (1976) 539.

[13] Gradshteyn, I. S. and Ryzhik, J. M., Table of Integrals, Series and Products (Academic Press, N.Y.) 1980.

[14] Dederichs, P. H., Zeller, R. and Schroeder, K., Point Defects in Metals II (Springer-Verlag, Berlin) 1980.

[15] Hansen, E. R., A Table of Series and Products (Prentice Hall, New Jersey) 1975. 